\title{
Cobertura de inmunización contra el virus de la hepatitis B en hijos nacidos de madres portadoras de la infección. Huanta, Ayacucho, 2014-2018 Immunization coverage against the hepatitis B virus in children from chronic carrier mothers. Huanta, Ayacucho, 2014-2018
}

\author{
Luis Crispin-Huamani ${ }^{1,3, a}$, César Cabezas ${ }^{1,2, b}$, Andrea Sucari-Idrogo ${ }^{1,0}$ \\ ${ }^{1}$ Facultad de Medicina, Universidad Nacional Mayor de San Marcos. Lima, Perú. \\ ${ }^{2}$ Centro Nacional de Salud Pública, Instituto Nacional de Salud. Lima, Perú. \\ ${ }^{3}$ Asociación para el Desarrollo de la Investigación Estudiantil en Ciencias de la Salud, Universidad Nacional Mayor de San Marcos. Lima, Perú. \\ ${ }^{a}$ Bachiller en medicina, ORCID: https://orcid.org/0000-0002-9044-9594 \\ ${ }^{b}$ Médico infectólogo tropicalista, ORCID: https://orcid.org/0000-0001-5120-0713 \\ ${ }^{\circ}$ Bachiller en medicina: https://orcid.org/0000-0002-0365-2894
}

\section{An Fac med. 2019;80(2):163-6 / DOI: https://10.15381/anales.802.16299}

\section{Correspondencia:}

Luis Javier Crispin Huamani

javito15810@gmail.com

Recibido: 19 de junio 2019

Aceptado: 26 de junio 2019

Publicación en línea: 28 de junio 2019

Conflictos de interés: Los autores declaran no tener conflictos de interés

Fuente de financiamiento

Autofinanciado

Contribuciones de autoría: CC ha participado en la concepción y diseño del artículo. CC, LCH y ASI contribuyeron con recolección de resultados y la redacción del artículo. CC participó en la revisión crítica del artículo. LCH y ASI contribuyeron con el análisis e interpretación de datos y asesoría estadística. Además CC, $\mathrm{LCH}$ y ASI aprobaron la versión final del artículo.

Citar como: Crispin-Huamani L, Cabezas C, Sucari-ldrogo A. Cobertura de inmunización contra el virus de la hepatitis $B$ en hijos nacidos de madres portadoras de la infección. Huanta, Ayacucho, 2014-2018. An Fac med. 2019;80(2):163-6.DOI: https// 10.15381/ anales.802.16299

\section{Resumen}

Introducción. En el marco del plan de eliminación de la infección por virus de la hepatitis B (HBV) es necesario conocer la cobertura de inmunización en los hijos de madres portadoras. Objetivo. Determinar el nivel de cobertura de inmunización en los hijos nacidos de madres portadoras de la infección por HBV en el Hospital de Huanta, Ayacucho, entre los años 2014 a 2018. Métodos. Se realizó un estudio observacional descriptivo. La población incluyó a los hijos nacidos de madres portadoras de la infección por HBV. Se utilizaron fuentes de información secundaria de registro de inmunización contra HBV. Resultados. Se registraron 90 madres portadoras de la infección por HBV cuyos hijos nacieron en el lugar de estudio. La cobertura total de inmunización contra HBV fue $64,47 \%$, la cobertura de la dosis de nacimiento de la vacuna fue $100 \%$ y la cobertura de aplicación de la inmunoglobulina antihepaitis B de 77,78\%. Conclusiones. La cobertura total de inmunización contra HBV se encuentra por debajo de lo esperado según las recomendaciones de la OMS.

Palabras clave: Hepatitis B; Cobertura de Vacunación; Portador; Transmisión Vertical de Enfermedad Infecciosa

Abstract

Introduction. In the framework of the plan for the elimination of infection with hepatitis B virus (HBV), it's necessary to know the immunization coverage in the children of carrier mothers. Objective. To determine the level of immunization coverage in children born to mothers with HBV infection at the Hospital de Huanta, Ayacucho, between 2014 and 2018. Methods. A descriptive observational study was carried out. The population included children born to mothers with HBV infection. Secondary information sources of immunization record against HBV were used. Results There were 90 mothers carrying the HBV infection whose children were born at the study site. The total immunization coverage against HBV was $64,47 \%$, the coverage of the birth dose of the vaccine was $100 \%$ and the coverage of application of immunoglobulin antihepaitis B of $77,78 \%$. Conclusions. The total immunization coverage against HBV is below what was expected according to WHO recommendations.

Keywords: Hepatitis B; Vaccination Coverage; Carrier State; Infectious Disease Transmission, Vertical 


\section{INTRODUCCIÓN}

A nivel mundial existen aproximadamente 257000000 individuos que sufren de un cuadro crónico causado por virus de la hepatitis $B$ (HBV), el cual ocasiona alrededor de 780000 muertes cada año por sus complicaciones como cirrosis hepática o cáncer de hígado ${ }^{1,2,3}$. El riesgo de desarrollar cronicidad es mayor para aquellos que adquieren la infección al nacimiento o hasta los 6 primeros meses de edad. La cronicidad por HBV se desarrolla en aproximadamente $90 \%$ de los que adquirieron la infección antes de cumplir los 12 meses de edad, en el 25$50 \%$ entre 1 a 5 años y en el 5-10\% después de los 5 años $^{4}$.

Persiste una carga sustancial de infección crónica por HBV debido a que la cobertura global de vacunación con la dosis al nacer sigue siendo baja. Si bien se ha incrementado en los últimos años gracias a las políticas implantadas en los diferentes países, parece aún insuficiente ${ }^{2}$. Se han logrado reducciones significativas en la prevalencia de la infección, gracias al impacto de los programas de inmunización contra el HBV6; así, dos estudios en menores de 5 años que habitaban en zonas de alta prevalencia en nuestro país, mostraron reducciones de la infección por HBV de un $83 \%$ y $92 \%$, respectivamente ${ }^{7,8}$.

En el Perú, en el año 2003 se instauró la vacunación universal contra el HBV, la cual actualmente se aplica al nacimiento, y luego a los dos, cuatro y seis meses-mediante la vacuna pentavalente. Dicha vacuna es de tipo recombinante y presenta una protección de aproximadamente 95\%. El Ministerio de Salud determina como punto de referencia de la cobertura de la vacuna al nacimiento en un $79 \%$ y en $95 \%$ como meta ideal para cada región $n^{5}$.

Las investigaciones en nuestro país acerca de la implementación de la vacuna contra el HBV muestran que alcanzó una cobertura de alrededor de $80 \%$ entre 2010 a $2015^{6}$. Dichos estudios evaluaron de forma general a toda la población, mas no a poblaciones de riesgo ${ }^{5}$; además, no se ha tomado en cuenta la co- bertura de las 3 dosis de la vacuna contra el HBV incluidas en la vacuna pentavalente y la administración de inmunoglobulina humana en los posibles casos de trasmisión vertical (madre-hijo). A pesar de que la vacunación universal contra HBV se implantó en las regiones de América Latina, existe escasez de información sobre la calidad de proceso de vacunación y su impacto en la trasmisión de HBV en toda la región, sobre todo en zonas y poblaciones vulnerables como Huanta en la provincia de Ayacucho ${ }^{6}$. La Organización Panamericana de la Salud (OPS) considera la eliminación de la transmisión madre-hijo y la transmisión dentro de la niñez, como parte de la estructura hacia la disminución de la infección por HBV.

En el marco del plan de eliminación de la infección por HBV es necesario conocer los actuales datos sobre la cobertura de inmunización en la población y particularmente en la población infantil; ya que para que dicho plan sea viable, se debe contar con el menor número de susceptibles posibles, llevándose idealmente esta cifra a cero. Así, el objetivo del presente estudio fue determinar el nivel de cobertura de inmunización contra el HBV en los hijos nacidos de madres que portan la infección en el Hospital de Huanta, Ayacucho, entre los años 2014 y 2018.

\section{MÉTODOS}

Se realizó un estudio observacional de tipo descriptivo y retrospectivo. La población incluyó a los hijos nacidos de madres portadoras de la infección por HBV, cuyos nacimientos fueron registrados en el Hospital de Apoyo de Huanta, Ayacucho, entre enero de 2014 y diciembre de 2018. Se solicitó acceso a la información de registro de los sujetos de estudio en la oficina de Coordinación de Prevención y Control VIH-SIDA y Hepatitis B de la Unidad Ejecutora Red de Salud Ayacucho Norte sede Huanta. Además, se solicitó a la Oficina de Aseguramiento Público, perteneciente a dicha unidad, el acceso a la información sobre inmunización (lugar y fecha de aplicación) de la vacuna de recién nacido contra el virus de hepatitis $B$ (HBV V-n) y de las tres dosis de la vacuna pentavalente. En el libro de nacimientos del Servicio de Neonatología del Hospital de Huanta se encontró registrada la información de la aplicación de la HBV v-n y de la inmunoglobulina humana; además, la siguiente información perinatal: edad materna al momento del parto, edad gestacional al momento del parto, tipo de parto, sexo del recién nacido, peso al nacimiento, número de controles pre natales, lugar de nacimiento.

Se consignó y ordenó la información en una base de datos consolidada elaborada en una de cálculo de Microsoft Excel 2013 para poder realizar el análisis respectivo. Se obtuvieron medidas de frecuencia y porcentaje de las variables del estudio y se representaron los resultados en tablas de contingencia. El estudio fue aprobado por el Comité de Ética de la Facultad de Medicina de la Universidad Nacional Mayor de San Marcos. Solo se utilizaron fuentes de información secundaria, por lo que no fue necesario el uso de consentimiento informado. En todo momento se mantuvo la información recolectada bajo reserva y se preservó el anonimato de los participantes.

\section{RESULTADOS}

Según los registros analizados, existieron 93 madres portadoras de la infección por HBV cuyos hijos nacieron en el Hospital de Apoyo de Huanta, Ayacucho, entre los años 2014 a 2018. Se verificó y completó la información de nacimiento y variables perinatales con el registro del libro de nacimientos del Servicio de Neonatología del Hospital de Huanta, donde se ubicó a 90 hijos nacidos de madres portadoras de la infección por HBV. Se contrastó la información con la base de datos otorgada por la Oficina de Aseguramiento Público sobre inmunizaciones (HBV v-n y tres dosis de la vacuna pentavalente), en la cual no se logró ubicar a tres sujetos de estudio. Finalmente se contó con 2 sujetos de estudio con información sobre características perinatales, aplicación de la HBV $v-n$ y aplicación de la inmunoglobulina humana; y 88 sujetos de estudio con dicha información, además de información sobre la aplicación de las tres dosis de la vacuna pentavalente. 
En cuanto a la aplicación de la HBV v-n, se encontró una cobertura del 100\%. Pero se observó que sólo 44 sujetos de estudio $(48,88 \%)$ recibieron la vacuna dentro de las primeras 24 horas de nacimiento. Este cifra varía inversamente con el paso de los años ya que en el año 2014 un $60 \%$ de los recién nacidos recibieron la vacuna dentro de las primeras 24 horas de vida; en el año 2016 esa cifra llegaba al 76,47\%, pero para los siguientes años dicha cifra descendió, siendo para el año 2018 sólo de un 22,27\% tal como se muestra en la tabla 1.

En cuanto a la cobertura de la aplicación de la inmunoglobulina antihepatitis $B$, se evidenció que la cobertura de la misma fue de $77,78 \%$ de forma global. En el año 2014 fue de sólo un 40\%. Según se muestra en la tabla 2, el porcentaje fue ascendiendo a medida que pasaron los años: llegando a un $57,14 \%$ para el año 2015; a un 94,11\% para el año 2016; y a un $95,45 \%$ para el año 2018, su cifra máxima.

Se observó que existieron 84 sujetos que nacieron hasta el octubre del 2018, de los cuales $82(97,61 \%)$ recibieron la primera dosis de la vacuna pentavalente; 82 sujetos nacieron hasta agosto del 2018, de los cuales 77 (93,9\%) recibieron la segunda dosis de la vacuna pentavalente; y 76 sujetos de estudio nacieron hasta el junio del 2018, de los cuales 61 $(80,26 \%)$ recibieron la tercera dosis de la vacuna pentavalente, como se muestra en la tabla 3.

En la tabla 4 se muestra la cobertura total de inmunización en los 76 sujetos de estudio que contaban con la información completa de inmunizaciones (aplicación de la HBV v-n, aplicación de la

Tabla 1. Cobertura de aplicación de la dosis de nacimiento de la vacuna contra el HBV según año de nacimiento, en hijos de madres portadoras. Hospital de Huanta, Ayacucho, 2014-2018.

\begin{tabular}{lccc} 
Año & Si se aplicó la HBV v-n & No se aplicó la HBV v-n & Total \\
\hline 2014 & $6(60 \%)$ & $4(40 \%)$ & 10 \\
\hline 2015 & $13(61,90 \%)$ & $8(38,09 \%)$ & 21 \\
\hline 2016 & $13(76.47 \%)$ & $4(23,52 \%)$ & 17 \\
\hline 2017 & $7(35 \%)$ & $13(65 \%)$ & 20 \\
\hline 2018 & $5(22,27 \%)$ & $17(77,27 \%)$ & 22 \\
\hline Total & $44(48,88 \%)$ & $46(51,11 \%)$ & $90(100 \%)$ \\
\hline
\end{tabular}

HBV v-n: dosis de nacimiento de la vacuna contra el virus de Hepatitis B

IG: Inmunoglobulina inmunoglobulina humana y aplicación de las tres dosis de la vacuna pentavalente), observándose que 49 de ellos $(64,47 \%)$ cumplieron con la aplicación de los 5 componentes de inmunización.

\section{DISCUSIÓN}

Según la Norma Técnica de Manejo de la Hepatitis Viral B en el Perú 2018 del Ministerio de Salud, la transmisión del HBV de madre a niño se puede prevenir aplicando a los recién nacidos la inmunoglobulina humana y la HBV v-n durante las primeras 12 horas $^{9}$. Si bien la cobertura de la HBV v-n fue del $100 \%$, la información muestra que el $48,88 \%$ de los sujetos de estudio la recibieron dentro de las primeras 24 horas. No se cuenta con datos del porcentaje que recibió la vacuna en las primeras 12 horas.

En un revisión realizada por Lee y col., se encontró que la aplicación de la HBV y de la inmunoglobulina $\mathrm{v}-\mathrm{n}$ disminuyeron significativamente el riesgo de desarrollar la infección al compararla con el grupo de placebo/no intervención, ello con un $R R$ de 0,28 y 0,52 respectivamente,

Tabla 02. Cobertura de aplicación de la inmunoglobulina antihepaitis B según año de nacimiento, en hijos de madres portadoras. Hospital de Huanta, Ayacucho, 2014-2018.

\begin{tabular}{lccc} 
Año & Si se aplicó IG & No se aplicó IG & Total \\
\hline 2014 & $4(40 \%)$ & $6(60 \%)$ & $10(11,11 \%)$ \\
\hline 2015 & $12(57,14 \%)$ & $9(42,85 \%)$ & $21(23,33 \%)$ \\
\hline 2016 & $16(94,11 \%)$ & $1(5,88 \%)$ & $17(18,88 \%)$ \\
\hline 2017 & $17(85 \%)$ & $3(15 \%)$ & $20(22,22 \%)$ \\
\hline 2018 & $21(95,45 \%)$ & $1(4,54 \%)$ & $22(24,44 \%)$ \\
\hline Total & $70(77,78 \%)$ & $20(22,22 \%)$ & $90(100 \%)$ \\
\hline
\end{tabular}

un $\mathrm{p}$ de $95 \%$ en ambos casos y un intervalo de confianza de 0,20 a 0,40 y de 0,44 a 0,63 , respectivamente. Además, se observó que la combinación de ambas redujo significativamente el riesgo de desarrollar la infección con un RR de 0,08, un $\mathrm{p}$ de $95 \%$ y un intervalo de confianza de 0,03 a $0,17^{10}$.

Se evaluó la cobertura de las tres dosis de la vacuna pentavalente, indicadas en el esquema de vacunación nacional. La cobertura de la aplicación de la primera dosis fue de $97,61 \%$ y 67 de ellos contaban con información acerca de la edad de aplicación de la misma; 58 de ellos $(86,56 \%)$, recibieron la vacuna a la edad indicada en el programa de vacunación, es decir a los 2 meses. La cobertura de aplicación de la segunda dosis fue de 93,90\%, de ellos 59 contaban con información acerca de la edad de aplicación. De ese grupo, $47(79,66 \%)$ la recibieron a la edad esperada; es decir, a los 4 meses. La cobertura de la aplicación de la tercera dosis fue más baja aún, Ilegando solo a un $80,26 \%$, y el $87,80 \%$ de los 41 sujetos que contaban con información acerca de la edad de la aplicación de la misma la recibieron a los 6 meses de vida.

Si bien la dosis de nacimiento de la vacuna contra el HBV puede prevenir la infección en un porcentaje alto, cuando esta se asocia al uso de inmunoglobulina antihepatitis B y al cumplimiento de las siguientes dosis de aplicación de la vacuna contra el $\mathrm{HBV}$, reduce la tasa de infección a valores entre 0,7 a 1,1\%. Por lo que luego de completar la dosis de la misma, un $98 \%$ de infantes que nacen a término y sin patologías asociadas deberían tener concentraciones de anticuerpos adecuados para asegurar protección contra la infección por el HBV ${ }^{11}$. 
Tabla 3. Cobertura de aplicación de la primera, segunda y tercera dosis de la vacuna pentavalente, en hiijos de madres portadoras de HBV. Hospital de Huanta, Ayacucho, 2014-2018.

$$
\text { Ítem }
$$
Frecuencia (n) / Porcentaje (\%)

Aplicación de la primera dosis de la vacuna pentavalente

\begin{tabular}{cc}
\hline $\mathrm{Si}$ & $82(97,61 \%)$ \\
\hline No & $2(2,38 \%)$ \\
\hline Total & $84(100 \%)$ \\
\hline Aplicación de la segunda dosis de la vacuna pentavalente & \\
\hline $\mathrm{Si}$ & $77(93,90 \%)$ \\
\hline No & $5(6,09 \%)$ \\
\hline Total & $82(100 \%)$ \\
\hline Aplicación de la tercera dosis de la vacuna pentavalente & \\
\hline Si & $61(80,26 \%)$ \\
\hline No & $15(19,73 \%)$ \\
\hline Total & $76(100 \%)$ \\
\hline
\end{tabular}

Se concluye que existe un $64 \%$ de cobertura de inmunización contra el HBV en hijos nacidos de madres portadoras de la infección en el Hospital de Huanta, que comprende las cuatro dosis de la vacuna contra el HBV y la inmunoglobulina humana. El estudio únicamente incluyó información de los nacidos en el Hospital de Huanta y no necesariamente a todos los recién nacidos de la provincia, por lo

Tabla 4. Cobertura de inmunización contra el HBV en los 76 hijos de madres portadoras que tuvieron información completa de inmunizaciones: aplicación de la HBV v-n, aplicación de la inmunoglobulina humana y aplicación de las tres dosis de la vacuna pentavalente.

Ítem Frecuencia (n) / Porcentaje (\%)

Aplicación de vacunas

contra el HBV e

inmunoglobulina humana

\begin{tabular}{lc}
\hline $\mathrm{Si}$ & $49(64.47 \%)$ \\
\hline No & $27(35.52 \%)$ \\
\hline Total & $76(100 \%)$ \\
\hline
\end{tabular}

que sería pertinente realizar futuros estudios considerando también a los nacidos y/o registrados en los demás centros de salud con fuente de información preferentemente primaria y con seguimiento en dicha población. Es necesario garantizar la disponibilidad de recursos biológicos para la inmunización, así como una plataforma de información para un realizar el seguimiento adecuado y uso de las normas establecidas para la inmunización de los recién nacidos. Se debe seguir fomentando un trabajo conjunto entre la población y el sector salud a través de intervenciones en gestantes y recién nacidos para la prevención de la transmisión madre-hijo del HBV en el Perú.

\section{AGRADECIMIENTOS}

Al personal de la Unidad Ejecutora Red de Salud Ayacucho Norte sede Huanta y al Servicio de Neonatología del Hospital de Apoyo de Huanta por su colaboración y apoyo al presente estudio mediante la accesibilidad a sus ambientes con la finalidad de recabar la información solicitada.

\section{REFERENCIAS BIBLIOGRÁFICAS}

1. Organización Mundial de la Salud [Internet]. La prevención de la transmisión perinatal del virus de la hepatitis B: guia para introducir y fortalecer la administración de la dosis de vacuna contra la hepatitis B al nacer, 2017 [Fecha de acceso: 15 de enero 2019]. Disponible en: https://apps.who. int/iris/handle/10665/254645

2. World Health Organization [Internet]. Global hepatitis report, 2017 [Fecha de acceso: 9 de enero 2019]. Disponible en: https://apps.who.int/iris/ bitstream/handle/10665/255016/9789241565455eng.pdf?sequence $=1$

3. World Health Organization [Internet]. Hepatitis B, 2019 [Fecha de acceso: 15 de enero 2019]. Disponible en: https://www.who.int/en/news-room/ fact-sheets/detail/hepatitis-b

4. Organización Mundial de la Salud. Vacunas contra la hepatitis B: documento de posición de la OMS, julio de 2017. Wkly Epidemiol Rec. 2017;92(27):369-92.

5. Borda-Olivas A, Florián A, Montalvan E, Dedios M Cabezas C, Donaires F. Cumplimiento de la vacunación contra el virus de la hepatitis $B$ en recién nacidos de hospitales de Lima y Callao. Rev Peru Med Exp Salud Pública. 2018;35(3):465-70. DOI: 10.17843/rpmesp.2018.353.3619

6. Ropero A, Perez-Villar S, Pacis-Tirso C, Contreras M, El Omeiri N, Ruiz-Matus C. Progress in vaccination towards hepatitis $B$ control and elimination in the Region of the Americas. BMC Public Health. 2017; 17: 325. DOI: 10.1186/s12889-017-4227-6

7. Cabezas C, Ramos F, Vega M, Suárez M, Romero G, Carrillo C, et al. Impact of the immunization program integrated to the expanded immunization program (Epi) in Huanta,1994-1997. Rev Gastroenterol Peru. 2000;20(3):201-212.

8. Cabezas-Sanchez C, Trujillo-Villarroel O, ZavaletaCortijo C, Culqui-Lévano D, Suarez-Jara M, CuevaMaza N. Prevalence of hepatitis B infection in children under 5 years old on indigenous communities of the Peruvian Amazonia after immunization interventions. Rev Peru Med Exp Salud Publica. 2014;31(2):204-210.

9. Plataforma Digital Única del Estado Peruano [Internet]. Norma Técnica de salud para la prevención, diagnóstico y tratamiento de la Hepatitis Viral B en el Perú, 2018 [Fecha de acceso: 20 de enero 2019]. Disponible en: https://www.gob.pe/institucion/ minsa/normas-legales/235349-1330-2018-minsa

10. Lee C, Gong Y, Brok J, Boxall EH, Gluud C. Hepatitis $B$ immunization for newborn infants of hepatitis B surface antigen-positive mothers. Cochrane Database Syst Rev. 2006;(2): CD004790. DOI: 10.1002/14651858.CD004790.pub2

11. Mast EE, Margolis HS, Fiore AE, Brink EW, Goldstein ST, Wang SA, et al. A comprehensive immunization strategy to eliminate transmission of hepatitis B virus infection in the United States: recommendations of the Advisory Committee on Immunization Practices (ACIP) part 1: immunization of infants, children, and adolescents. MMWR Recomm Rep. 2005;54(RR-16):1-31. 